\title{
Terahertz Time-Domain Spectroscopy and Imaging
}

\section{Yao-Chun Shen*}

Department of Electrical Engineering and Electronics, University of Liverpool, Liverpool L69 3GJ, UK

Terahertz $(\mathrm{THz})$ waves are a form of electromagnetic wave, like gamma-rays, $\mathrm{X}$-rays, ultraviolet light, visible light, infrared light, radio and microwaves (Figure 1). On the frequency spectrum, the $\mathrm{THz}$ region of the electromagnetic spectrum spans the frequency range between the microwave and the mid-infrared $(300 \mathrm{GHz}-30 \mathrm{THz})$. The center portion of the $\mathrm{THz}$ region offers an unique combination of many remarkable properties: (1) like infrared radiation, $\mathrm{THz}$ radiation gives rise to individual 'fingerprints' spectra for many crystalline materials, making $\mathrm{THz}$ spectroscopy a useful tool for material characterization; (2) like microwave, $\mathrm{THz}$ radiation can penetrate deep into most polymer and clothing materials, thus $\mathrm{THz}$ imaging can be used to map internal structures of a sample; (3) it is safe to use $\mathrm{THz}$ radiation as its photon energy is millions of times smaller than that of X-rays. The combination of these properties makes $\mathrm{THz}$ technology a potentially very powerful technique for a range of imaging and spectroscopy applications. However, historically the exploitation of this $\mathrm{THz}$ spectral region has been difficult owing to the lack of suitable source and detector. There has therefore been considerable interest in the development of $\mathrm{THz}$ technology, in particular when considering that the technology development of its neighboring spectral regions (e.g., optical and microwave frequency) has resulted in the birth of new industrials.

With the advances in material research and ultrafast laser technology, the past twenty years have seen a revolution in $\mathrm{THz}$ devices and systems [1-4]. Of particular significance is the development and commercialization of $\mathrm{THz}$ time-domain spectroscopy (THz-TDS) and $\mathrm{THz}$ time-domain imaging (THz-TDI) systems. The core technology

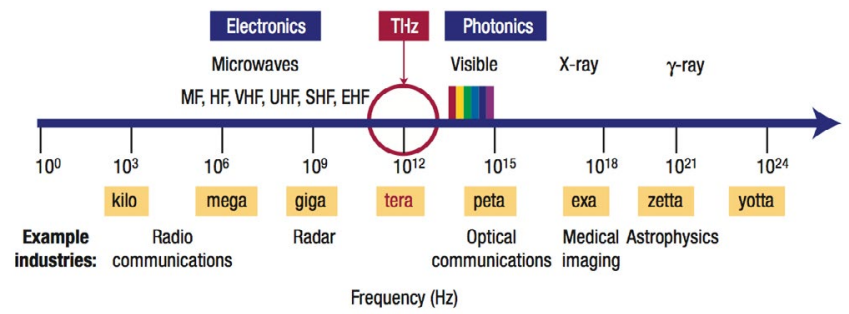

Figure 1: Schematic diagram showing the location of $\mathrm{THz}$ band in the electromagnetic spectrum (reproduced from [1]).

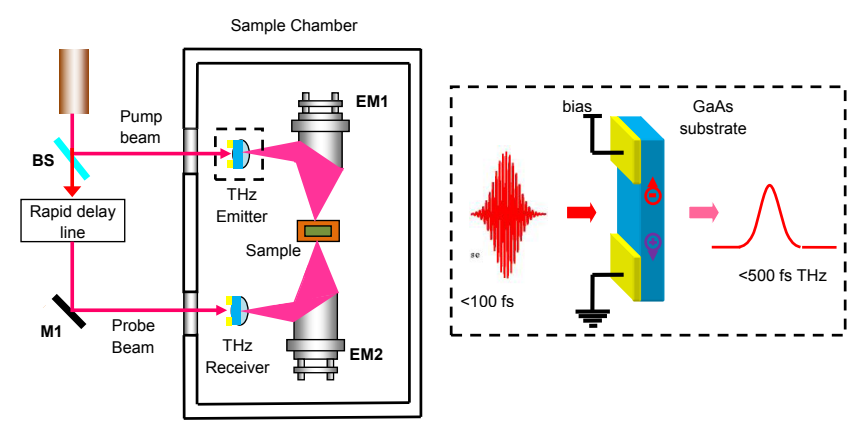

Figure 2: Left: Schematic diagram of a THz-TDS instrument. BS: beam splitter; M1: metallic mirror; OEM1-OEM2: off-axis elliptic mirrors. Right: Generation of broadband terahertz pulses in a Gallium Arsenide (GaAs) photoconductive antenna (reproduced from [2]) behind both THz-TDI and THz-TDS is the coherent generation and detection of short pulses of broadband $\mathrm{THz}$ radiation by using ultrafast femtosecond laser. To date, the most commonly used device for the generation of ultrashort $\mathrm{THz}$ pulses is photoconductive antenna [5]. In this technique, a biased photoconductive antenna $(\mathrm{THz}$ emitter antenna) is illuminated using an above-band gap femtosecond pulse of near-infrared light (Figure 2). Electron-hole pairs are generated in GaAs semiconductor crystal, and these photo-generated carriers are then accelerated by the applied electric field. The physical separation of the electrons and holes forms a macroscopic space-charge field oriented opposite to the biasing field, and thus, the total net electrical field undergoes rapid changes. The fast temporal change in electric field produces a transient current, which generates a pulse of electromagnetic radiation in the $\mathrm{THz}$ frequency range. The photoconductive antenna can also be used for the detection of the THz pulse. In contrast to the $\mathrm{THz}$ emitter antenna, the $\mathrm{THz}$ detector antenna is not biased. The photo-generated carriers are actually driven by the electric field of the incident $\mathrm{THz}$ pulse, and the resultant photocurrent is proportional to the time integration of the product of the incident $\mathrm{THz}$ electric field and the total number of photo-generated carriers in the photoconductive antenna. In the limit of an ideal photoconductive antenna with carrier lifetime much shorter than the duration of the THz pulse, the detected photocurrent is directly proportional to the $\mathrm{THz}$ electric field strength [6].

Figure 2 shows the schematic diagram of a typical THz-TDS instrument. In brief, a beam splitter separated the near-infrared light into two beams: an excitation beam and a probe beam. The excitation beam is used to pump a $\mathrm{THz}$ emitter antenna for generating a short pulse of broadband $\mathrm{THz}$ radiation. The generated $\mathrm{THz}$ pulse is focused and transmitted through the sample, before being detected using a $\mathrm{THz}$ receiver antenna which is gated by the probe beam from the same ultrafast laser system. In this way, the time-resolved electric field of the $\mathrm{THz}$ pulse could be recorded by scanning the time-delay between the $\mathrm{THz}$ pulse and the near-infrared probe beam using a variable delay stage. For quantitative spectral measurement, the time-resolved electric field of $\mathrm{THz}$ pulses before and after propagating through a sample is measured. The THz pulse transmitted through the sample is modified by the dispersion and absorption of the media under examination. Both the absorption coefficient and the refractive index of the sample could be extracted from the measured $\mathrm{THz}$ signal without the need for the Kramers-Kronig transformation [3].

* Corresponding author: Yao-Chun Shen, Department of Electrical Engineering and Electronics, University of Liverpool, Liverpool L69 3GJ, UK, Tel: 44-151-794-4575; Fax: 44-151-794-4540; E-mail: y.c.shen@liverpool.ac.uk

Received December 09, 2013; Accepted December 11, 2013; Published December 13, 2013

Citation: Shen YC (2013) Terahertz Time-Domain Spectroscopy and Imaging. J Electr Electron Syst 3: e113. doi:10.4172/2332-0796.1000e113

Copyright: $\odot 2013$ Shen YC, et al. This is an open-access article distributed under the terms of the Creative Commons Attribution License, which permits unrestricted use, distribution, and reproduction in any medium, provided the original author and source are credited. 
This coherent generation and detection of pulsed $\mathrm{THz}$ radiation offers two remarkable advantages. Firstly, this technology directly measures the transient electric field, not simply the intensity of the $\mathrm{THz}$ radiation. This yields $\mathrm{THz}$ spectrum with far better signal to noise ratio and dynamic range as compared with the Fourier Transform Infrared Spectroscopy (FTIR) method. High quality $\mathrm{THz}$ spectrum is now routinely obtained in less than $20 \mathrm{~ms}$ without the need for cryogen cooled bolometer, making $\mathrm{THz}$ spectroscopy more easily and widely accessible. Secondly, the use of pulsed radiation and the associated coherent detection scheme preserves the time-gated phase information, upon which $\mathrm{THz}$ imaging has been developed for characterizing the internal structures of a sample quantitatively and non-destructively.

THz spectroscopy technology-spectral fingerprints: Absorption features within the mid-infrared region are dominated by intramolecular vibrations of sample molecules thus mid- infrared spectral features are "molecule fingerprints". In contrast, absorption features in $\mathrm{THz}$ region are dominated by inter-molecular vibrations, corresponding to motions associated with coherent, delocalized movements of large numbers of atoms and molecules $[7,8]$. Such collective phonon modes only exist in materials with periodic structure. In this sense, $\mathrm{THz}$ spectral features are "crystal fingerprints", and materials with identical molecular structures but different crystal forms (so-called crystal polymorphs) are expected to have different $\mathrm{THz}$ spectrum. Consequently $\mathrm{THz}$ spectroscopy is an excellent technique for characterizing the crystalline properties of solid materials, as the phonon lattice modes are probed directly. The spectral fingerprints at $\mathrm{THz}$ frequency range have been used for characterizing Active Pharmaceutical Ingredients (API), monitoring of dynamic process of co-crystal formation and solid phase transformations, detection and identification of explosives, to name a few [8-11].

THz imaging technology-imaging at depth: many common polymer and clothing materials are semi-transparent to $\mathrm{THz}$ radiation therefore $\mathrm{THz}$ radiation will be able to penetrate into a sample. At each interface or change in refractive index, portion of the $\mathrm{THz}$ radiation will be reflected/scattered back. By sending a pulse of broadband $\mathrm{THz}$ radiation into a sample and recording the reflected/scattered $\mathrm{THz}$ radiation as a function of time, one can map the inner structures and compositions of the sample. A number of imaging applications have been reported in areas as diverse as medical diagnosis of human tissue, analysis of polymer samples, industry sensor for fault detection of integrated circuit packaging in semiconductors and online and offline tablet inspection in the pharmaceutical industry among others [12-15]. Other promising applications of $\mathrm{THz}$ technology include general nondestructive test, $\mathrm{THz}$ metamaterial devices and $\mathrm{THz}$ electronics for energy harvesting $[1-4,16,17]$.

In summary, the enormous inherent potential of the THz technology led to a rapid development of $\mathrm{THz}$ systems, and the availability of commercial $\mathrm{THz}$ products has opened up many exciting opportunities in academic research and industrial application. In this article I have introduced the fundamentals and main attractive features of the $\mathrm{THz}$ time-domain technology and some of its promising application areas. Hope that this will raise the awareness of the emerging opportunities and usefulness offered by this exciting technology as in my view $\mathrm{THz}$ technology has the potential to play a significant role in all these areas.

\section{References}

1. Ferguson B, Zhang XC (2002) Materials for terahertz science and technology Nature Materials 1: 26-33.

2. Shen YC (2011) Terahertz pulsed spectroscopy and imaging for pharmaceutical applications: A review. Int J Pharm 417: 48-60

3. Jepsen PU, Cooke DG, Kock M (2011) Terahertz spectroscopy and imaging Modern techniques and applications. Laser \& Photonics Reviews 5: 124-166.

4. Zeitler JA, Shen YC (2013) Industrial Applications of Terahertz Imaging Terahertz Spectroscopy and Imaging, Springer Berlin Heidelberg, Germany.

5. Auston DH, Cheung KP, Smith PR (1984) Picosecond Photoconducting Hertzian Dipoles. Appl Phys Lett 45: 284-286.

6. Shen YC, Upadhya PC, Beere HE, Linfield EH, Davies AG, et al. (2004) Generation and detection of ultrabroadband terahertz radiation using photoconductive emitters and receivers. Appl Phys Lett 85: 164-166.

7. Walther M, Fischer BM, Ortner A, Bitzer A, Thoman A, et al. (2010) Chemical sensing and imaging with pulsed terahertz radiation. Analytical and Bioanalytical Chemistry 397: 1009-1017.

8. Jepsen PU, Clark SJ (2007) Precise ab-initio prediction of terahertz vibrational modes in crystalline systems. Chem Phys Lett 442: 275-280.

9. Nguyen KL, Frisc T, Day GM, Gladden LF, Jones W (2007) Terahertz time domain spectroscopy and the quantitative monitoring of mechanochemical cocrystal formation. Nature Materials 6: 206-209.

10. Zeitler JA, Newnham DA, Taday PF, Threlfall TL, Lancaster RW, et al. (2006) Characterization of temperature induced phase transitions in the five polymorphic forms of sulfathiazole by terahertz pulsed spectroscopy and differential scanning calorimetry. J Pharm Sci 95: 2486-2498.

11. Shen YC, Lo T, Taday PF, Cole BE, Tribe WR, et al. (2005) Detection and identification of explosives using terahertz pulsed spectroscopic imaging. Appl Phys Lett 86: 241116-241116-3.

12. Pickwell E, Wallace VP (2006) Biomedical applications of terahertz technology. J Phys D Appl Phys 39: R301-R310.

13. Ho L, Müller R, Römer M, Gordon KC, Heinämäki J, et al. (2007) Analysis of sustained-release tablet film coats using terahertz pulsed imaging. J Contro Release 119: 253-261.

14. May RK, Evans MJ, Zhong S, Warr I, Gladden LF, et al. (2011) Terahertz in-line sensor for direct coating thickness measurement of individual tablets during film coating in real-time. J Pharm Sci 100: 1535-1544.

15. Cai Y, Wang Z, Dias R, Goyal D (2010) Electro optical terahertz pulse reflectometry - an innovative fault isolation tool. Electronic Components and Technology Conference, Las Vegas, NV, USA.

16. Chen HT, Padilla WJ, Zide JMO, Gossard AC, Taylor AJ, et al, (2006) Active terahertz metamaterial devices. Nature 444: 597-600.

17. Hall S, Mitrovic IZ, Sedghi N, Shen YC, Huang Y, et al. (2013) Harvesting Using $\mathrm{THz}$ Electronics. Functional Nanomaterials and Devices for Electronics, Sensors and Energy Harvesting. 